Pierre-Alain Duc, Jonathan Braine and Elias Brinks, eds.

\title{
VLA HI and OVRO CO Interferometry of a Tidal Dwarf Galaxy
}

\author{
Elias Brinks \\ INAOE, Apdo. Postal 51 \& 216, Puebla, Pue 72000, Mexico \\ Pierre-Alain Duc \\ Service d'Astrophysique, CEA/DSM/DAPNIA, Saclay, F-91191, France
}

Fabian Walter

NRAO-AOC, P.O. Box O, Socorro, NM 87801, USA

\begin{abstract}
.
We present high resolution interferometric observations of the cool atomic and cold molecular ISM of the TDG candidate Arp 245N, an object resembling a dwarf galaxy in the northern tidal tail of the interacting system NGC 2992/3. We observed the HI line with the NRAO VLA and the $\mathrm{CO}(1 \rightarrow 0)$ transition with the OVRO millimeter interferometer at $5^{\prime \prime}-6^{\prime \prime}$ angular resolution ( $750 \mathrm{pc}$ linear resolution). These datacubes offer the required spatial and velocity resolution to determine whether the mass concentration near the tip of the tail is a genuine feature, and hence a good TDG candidate, or an artefact caused by a fortuitous alignment of our line of sight with the direction of the tail. A preliminary analysis seems to confirm that Arp $245 \mathrm{~N}$ is a self-gravitating entity.
\end{abstract}

\section{Introduction}

Tidal Dwarf Galaxies (TDGs) are objects resembling actively star forming dwarf galaxies and are assembled from the debris (tidal tails and bridges) launched into the IGM by violent galaxy interactions in which at least one member is a gas-rich galaxy. They are composed of stars and gas from the outskirts of one or both of the parent galaxies involved in the interaction. The recent surge of interest in TDGs started with papers by Mirabel, Lutz, \& Maza (1991) on the Superantennae and Mirabel, Dottori, \& Lutz (1992) on the Antennae (see also the review by Duc \& Mirabel 1999). Several groups of authors have since embarked on the exciting topic of TDGs as witnessed by these proceedings (see e.g., the contributions by Duc et al. and by Braine et al. this volume).

Currently outstanding questions are: (i) Are TDGs really recycled objects made of collisional debris or pre-existing galaxies involved in a three-body interaction? (ii) Are TDG genuine density enhancements in the tidal tails or are they merely due to projection effects along the line of sight? (iii) Do TDGs form self gravitating entities or are they simple transient condensations? (iv) Are TDGs 
Dark Matter (DM) dominated, like galaxies in general, and dwarf galaxies in particular, or are they nearly devoid of DM, as theory predicts? (v) Finally do TDGs leave the potential well of their progenitors and hence constitute a sizeable fraction of the known dwarf galaxy population or do they eventually fall back and merge, leaving no trace?

All these questions have actually been raised for the particular TDG candidate identified in the northern tidal tail of Arp 245, an interacting system composed of two spiral galaxies, NGC 2992 and NGC 2993. Although Arp 245N was observed at many wavelengths and is one of the best studied TDG candidates, its nature as a tidal object or as a real entity have been challenged. Smith \& Struck (2001) argued that TDG Arp 245N could actually be a preexisting edge-on disk galaxy that is interacting with the other two galaxies. Hibbard et al. (this volume) point out that this system is viewed from an unfavorable perspective, making the projection effects particularly severe.

The combination of high-resolution kinematical and morphological data is critical to tackle all these issues. We have therefore carried out $\mathrm{HI}$ and $\mathrm{CO}$ interferometric observations of the system.

\section{The Interacting System Arp 245 (= NGC 2992/3)}

NGC 2992/3 is a relatively nearby system, at an adopted distance of $31 \mathrm{Mpc}$ $\left(\mathrm{V}_{\text {sys }}=2311 \mathrm{kms}^{-1}\right)$. Its prominent northern tidal tail hosts a tidal dwarf galaxy candidate which because of its proximity can be studied in detail (Fig. 1). The system was observed by us in HI with the NRAO $^{1}$ Very Large Array (VLA) at an intermediate angular resolution of $19^{\prime \prime} \times 14^{\prime \prime}$ (Duc et al. 2000).

Toward NGC 2992, the HI shows a peak at the location of the tidal dwarf candidate located at the tip of the tidal tail emanating from NGC 2992. HI is seen in absorption against the radio continuum from the nucleus. NGC 2992 is classified as a Seyfert 1.9 galaxy and in the light of $\mathrm{H} \alpha$ a biconical structure can be seen which extends out into the halo. Toward NGC 2993, the HI map has a ringlike structure which in its western extension has no optical counterpart. Within NGC 2993 and Arp 245N, HII regions are concentrated within HI clumps and trace star-forming regions. Numerical simulations of the NGC 2992/3 collision indicate that we see the system $\sim 100 \mathrm{Myr}$ after closest approach (Duc et al. 2000).

As reported by Braine et al. (2000, 2001), Arp 245 was detected with the IRAM $30^{-} \mathrm{m}$ dish in both the $\mathrm{CO}(1 \rightarrow 0)$ and the $\mathrm{CO}(2 \rightarrow 1)$ transitions. The observations also revealed that the $\mathrm{CO}$ emission is extended along the TDG. The $\mathrm{H}_{2}$ mass, assuming the standard (Galactic) $\mathrm{CO}$ to $\mathrm{H}_{2}$ conversion factor of $\mathrm{N}_{\mathrm{H}_{2} / \mathrm{I}_{\mathrm{CO}}}=2 \times 10^{20} \mathrm{~cm}^{-2} / \mathrm{K} \mathrm{km} \mathrm{s}^{-1}$ is $\sim 1.5 \times 10^{8} \mathrm{M}_{\odot}$.

An oxygen abundance of $12+\log (\mathrm{O} / \mathrm{H})=8.6$ was measured in the HII regions of the TDG candidate. This high metallicity excludes the hypothesis that the TDG is in fact a preexisting dwarf galaxy. It is also unlikely that a more massive galaxy was directly involved in the interaction. Indeed, the morphology of the

\footnotetext{
${ }^{1}$ The National Radio Astronomy Observatory is a facility of the National Science Foundation operated under cooperative agreement by Associated Universities, Inc.
} 


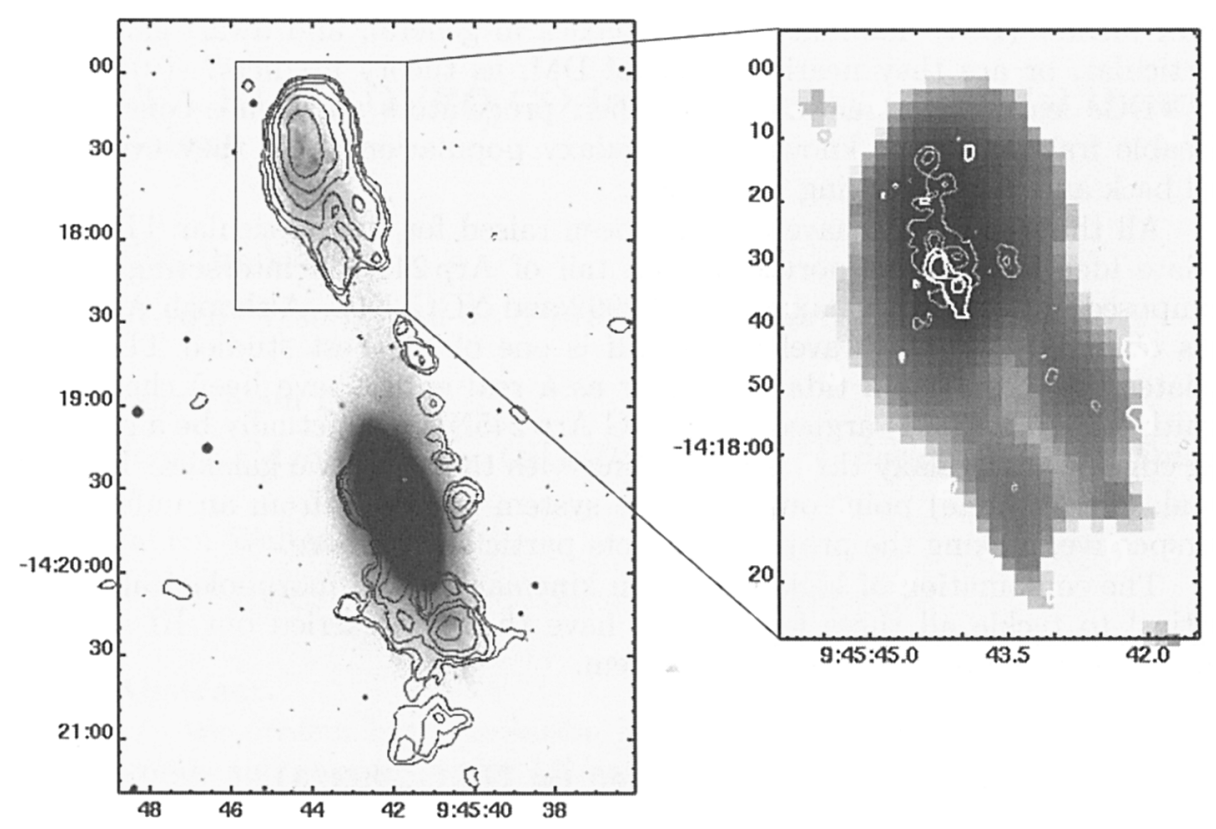

Figure 1. Left: Optical R-band image of NGC 2992 with overlaid HI contours from the high resolution VLA B-array observations. Right: Grey scale representation of the HI data centred on Arp 245N with superimposed the $\mathrm{H} \alpha$ emission (thin contours) and the high resolution OVRO $\mathrm{CO}(1-0)$ (thick contours). Coordinates are for a J2000.0 equinox.

system matches very well that of the numerical model in which only two galaxies are interacting.

\section{Observations}

\subsection{HI Observations}

The field containing the interacting pair NGC 2992/3 was observed with the VLA in its B-configuration on 24 April and 7/8 May 2001 for a total of almost 10 hours. Data calibration and reduction followed standard procedures using the Classic AIPS data reduction package. The final data products have a resolution of $6^{\prime \prime}$ (at $5 \mathrm{~km} \mathrm{~s}^{-1}$ velocity resolution) and reach an rms noise of $0.5 \mathrm{mJy}$ beam ${ }^{-1}$ per channel.

\subsection{CO Observations}

We observed the tidal dwarf near NGC 2992 in the $\mathrm{CO}(1 \rightarrow 0)$ transition using the Owen's Valley Radio Observatory's mm array (OVRO) in the E, C and L configurations for a total of 9 tracks from October 2001 through May 2002. 


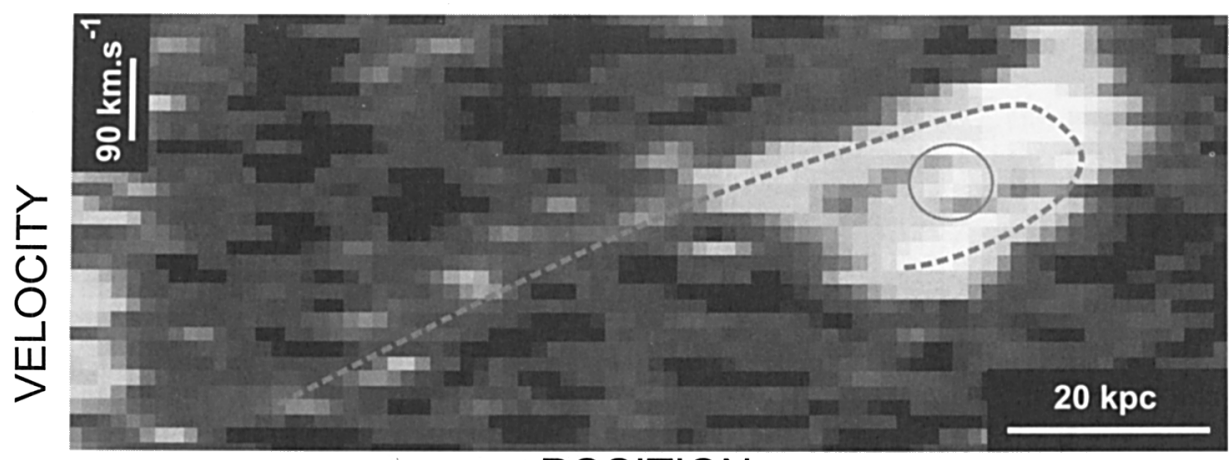

\section{POSITION}

Figure 2. Position-velocity diagram taken through the high resolution HI data along a band connecting NGC 2992 and Arp 245N, along the northern tidal tail.

The equatorial $\mathrm{E}$ configuration was needed to improve the beam shape for this $\delta=-14^{\circ}$ source. About 40 hours were spent on source. Data were recorded using a correlator setup resulting in velocity resolutions of $5 \mathrm{~km} \mathrm{~s}^{-1}$ (after online Hanning smoothing) with a total bandwidth of $320 \mathrm{~km} \mathrm{~s}^{-1}$. A datacube was produced using the MIRIAD software package which was CLEANed to a level of about twice the rms noise (noise: $16 \mathrm{mJybeam}^{-1}$ in a $5 \mathrm{~km} \mathrm{~s}^{-1}$ wide channel). The final beam size is $7.2^{\prime \prime} \times 4.0^{\prime \prime}$.

\section{Results}

Figure 1 shows an integrated HI map of the B-array VLA data only. It should be noted that an interferometer acts as a spatial filter and that the B-array is sensitive to structures with typical sizes of between $5^{\prime \prime}$ and $120^{\prime \prime}$. As a result, applying a CLEAN algorithm can leave some low level striping which will disappear once the new data are incorporated with the intermediate resolution maps.

The right panel in Figure 1 shows as contours overlaid on the HI map, the $\mathrm{CO}$ and $\mathrm{H} \alpha$ emission. $\mathrm{CO}$ is clearly detected in the OVRO observations and is found to be resolved. The total integrated flux is $\sim 3 \mathrm{Jy} \mathrm{km} \mathrm{s}^{-1}\left(\sim 10 \mathrm{~K} \mathrm{~km} \mathrm{~s}^{-1}\right)$, corresponding to a total molecular gas mass, assuming a standard conversion factor of $\mathrm{N}_{\mathrm{H}_{2} / \mathrm{I}_{\mathrm{CO}}}=2 \times 10^{20} \mathrm{~cm}^{-2} / \mathrm{K} \mathrm{km} \mathrm{s}^{-1}$, of $\mathrm{M}_{\mathrm{H}_{2}} \sim 3.5 \times 10^{7} \mathrm{M}_{\odot}$. This is about $25 \%$ of the single dish flux which implies that most of the molecular gas is distributed smoothly across the region.

\section{Discussion and Summary}

Arp $245 \mathrm{~N}$ is a typical TDG candidate in the sense that it is a major HI concentration associated with recent star formation which resides near the tip of the tidal tail. Because the latter is seen close to edge-on, the question thus arises whether the apparent concentration is a genuine feature (Hibbard \& Mihos, this volume). Bournaud et al. (2003, 2004; see also the contributions by 


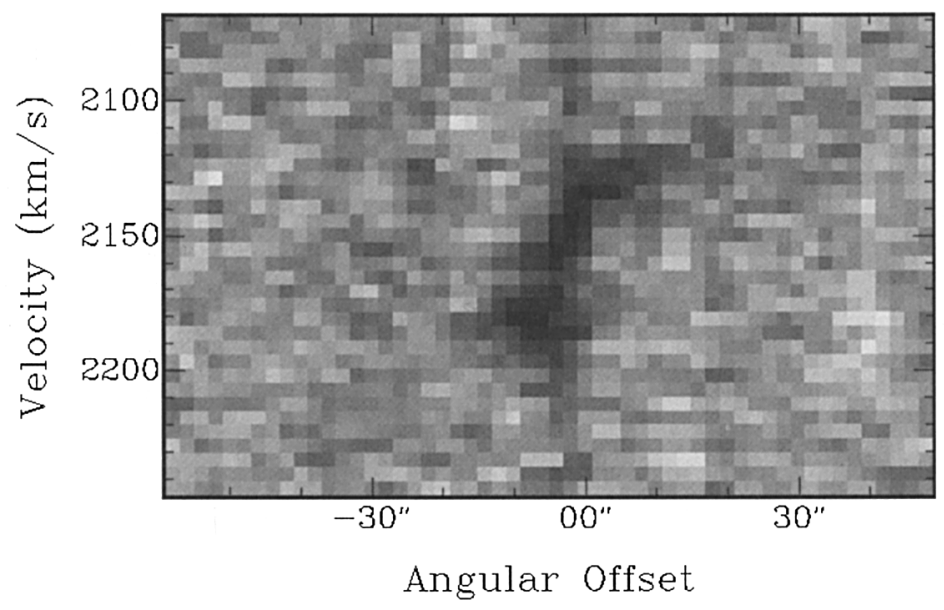

Figure 3. Position-velocity diagram taken through the high resolution HI data along a cross-cut through the TDG Arp 245N and perpendicular to the tidal tail. Angular distances along the cross-cut are in arcmin. The faint vertical stripe is an artefact (see text).

Amram et al., this volume) have run extensive numerical models and shown the characteristic shape in position-velocity space of a bona fide TDG and that of a spurious feature. They show that the kinematical signature of projection effects is a change in the sign of the velocity gradient along the tail before reaching its apparent tip. For curved tails that are extended enough in 3D space, a loop-like feature may even be seen in a position-velocity $(\mathrm{pV})$ diagram along the tails.

Figure 2 shows such a $\mathrm{pV}$ diagram along the tidal tail connecting the TDG candidate and NGC 2992 using the intermediate and high resolution VLA HI data. The signal was actually integrated over a band with a width similar to that of the tail. The loop expected for projection effects is clearly seen on the figure. The part of the tail that is seen curving back towards NGC 2992 (as seen projected on the sky) is actually consistent with our earlier numerical simulations of the system (Duc et al., 2000; see the face-on view in their Fig. 10).

The HI morphology of the tidal tail, as seen at high resolution in Fig. 1, may also give some clues as to the geometry of the system. Its U-like shape could be interpreted as being due to bending of the tidal tail near its apparent tip. The tail is actually not seen perfectly edge-on (as indicated, in the optical, by the large width of the stellar tail). Thanks to the higher spatial resolution provided by the VLA in its B-configuration, we can hence 'resolve' the projection effects - which was impossible with the early $\mathrm{C}$-array data. On this map, the HI column density seems to peak in the part of the tail which points back to NGC 2992. This is where OVRO detected the bulk of the molecular gas and where the brightest HII regions in the tail are found. The velocities of all these phases match. The spatial and velocity coincidence between the $\mathrm{CO}, \mathrm{H} \alpha$ and $\mathrm{HI}$ emission peaks at this location in the tail is a strong indication that a genuine 
condensation is present there and that this is likely the progenitor of a Tidal Dwarf galaxy.

At the same position, a $\mathrm{pV}$ diagram perpendicular to the tidal tail shows a small scale velocity gradient similar to that expected for a rotating body (see Fig. 3). The peak-to-peak velocity range is $100 \mathrm{~km} \mathrm{~s}^{-1}$. A word of caution is warranted here, though. As Duc et al. (2000) mentioned, the simulated pV diagram along the same direction in the numerical model shows a similar gradient. Further simulations are required to disentangle the embedded TDG from the rest of the tail. We should then be able to determine its dynamical mass and, comparing it with the luminous mass (corresponding to the HI condensation in the B-array), probe its dark matter content. Not taking into account the line of sight crowding, and considering all the matter present at the apparent tip of the tail, one derives a dynamical mass similar to the luminous one and equal to $\sim 2 \times 10^{9} \mathrm{M}_{\odot}$. For the above-mentioned reasons, these are most likely overestimates.

In summary, a first analysis of new high resolution $\mathrm{HI}$ and $\mathrm{CO}$ datacubes tends to support the existence of a bound entity within the northern tail of Arp 245. However, they also show the kinematical signature expected when part of the tail is bending away along the line of sight, and eventually back to NGC 2992. Because of these projection effects, the size and mass of the embedded TDG candidate derived from low resolution data are probably overestimates.

Acknowledgments. EB gratefully acknowledges a travel grant awarded by the IAU and partial financial support in the form of CONACyT grant No. 27606-E.

\section{References}

Bournaud, F., Duc, P.-A., \& Masset, F. 2003, A\&A, 411, L469

Bournaud, F., Duc, P.-A., Amram, P, \& Combes, F. 2004, submitted to A\&A

Braine, J., Duc, P.-A., Lisenfeld, U., Charmandaris, V., Vallejo, O., Leon, S., Brinks, E. 2001, A\&A, 378, 51

Braine, J., Lisenfeld, U., Duc, P.-A., Leon, S. 2000, Nature, 403, 867

Duc, P.-A., Brinks, E., Springel, V., Pichardo, B., Weilbacher, P., \& Mirabel, I. F. 2000, AJ, 120, 1238

Duc, P.-A. \& Mirabel, I. F. 1999, in IAU Symp. 186, Galaxy Interactions at Low and High Redshift, ed. J. E. Barnes \& D. B. Sanders (Dordrecht: Kluwer), p. 61

Mirabel, I.F., Dottori, H., \& Lutz, D. 1992, A\&A, 256, L19

Mirabel, I.F., Lutz, D., \& Maza, J. 1991, A\&A, 243, 367

Smith, B.J., \& Struck, C. 2001, AJ, 121, 710 